\title{
Adsorption of benzo(a)pyrene on to asbestos and manmade mineral fibres in an aqueous solution and in a biological model solution
}

\author{
P GERDE, P SCHOLANDER
}

From the Department of Chemical Engineering, Royal Institute of Technology, S-100 44 Stockholm, Sweden

ABSTRACT The adsorption of benzo(a)pyrene (BaP) on to three types of asbestos (chrysotile antophyllite, and amosite) and three types of manmade mineral fibres (MMMF) (rock wool, slag wool, and glass wool) in a physiological water solution was studied. Adsorption was determined from the decrease in the liquid concentration of $\mathrm{BaP}$ on the addition of the solid material. Results show that all the fibres weakly adsorb BaP, approximately within the same order of magnitude. The combined adsorption of $\mathrm{BaP}$ and phosphatidylcholine $(\mathrm{PC})$ on to chrysotile and amosite asbestos and on to rock wool in aqueous solution was also studied. PC, one of the major constituents in lung surfactant, forms a separate lipid phase in water consisting of micellar liposomes or lipid bilayers. A decrease in the liquid concentration of $\mathrm{PC}$ was found when any of the three materials was added, indicating adsorption of the lipid phase on to the fibres. A coincident decrease in the liquid concentration of $\mathrm{BaP}$ was also found indicating that $\mathrm{BaP}$ is readily solubilised in $\mathrm{PC}$ and will accompany the adsorption of this compound on to the fibres. Owing to the high lipid aqueous partition coefficient of $\mathrm{BaP}$, it is concluded that the direct adsorption of $\mathrm{BaP}$ on to the fibres will be negligible when $\mathrm{PC}$ is present in the system even at low concentrations. Phospholipid adsorption by the fibres and not their direct adsorption of aromatic hydrocarbons should therefore be the crucial parameter for this indirect interaction between fibres and aromatic hydrocarbons.

Exposure to asbestos and tobacco smoking have a synergistic effect on the risk of lung cancer. ${ }^{\prime}$ One hypothesis to explain this synergistic effect is that carcinogenic compounds in the tobacco smoke, in particular polycyclic aromatic hydrocarbons (PAHs), are adsorbed on to the surfaces of the asbestos fibres. This should increase both the retention time and the carcinogenic action of these substances in the lung. ${ }^{2}$ There is some concern that the same effect may occur with manmade mineral fibres (MMMF) that are now replacing asbestos in many areas. Apparently this adsorption can occur either in the ambient air as a gas phase process or after inhalation, preferably in the liquid phase of the extracellular lining layer of the lung. We have measured the adsorption of aromatic hydrocarbons on to asbestos and MMMF in the gas phase and have found it to be rather weak at typical ambient air concentrations (P Gerde, $P$ Scholander, IARC Conference 1987). This paper deals only with liquid phase adsorption. If fibres and PAHs, adsorbed

Accepted 10 March 1988 on to their typical carrier particles, are transported independently into the lung then the most plausible location for an interaction is in the lining layer of the bronchial tree. It is here that both agents have their densest deposition after inhalation, ${ }^{34}$ and most lung cancers connected with smoking and exposure to asbestos are of bronchial origin. ${ }^{\prime}$

The main composition of the bronchial lining layer has been reported to be $2-3 \%$ mucus glycoproteins, $0.3-0.5 \%$ lipids, $0 \cdot 1-0.5 \%$ proteins, and about $95 \%$ water. ${ }^{5}$ Recent investigations have shown that the lipids constitute a continuous layer on top of the lining layer from the alveolar region up to the trachea. ${ }^{6}$ Moreover, the lipids either form separate laminar layers or are dispersed as micellar liposomes within the aqueous phase. Owing to the great difference in the solubilities of PAHs between these two phases, this heterogeneity of the bronchial lining layer has to be considered. PAHs have low solubilities in water ${ }^{7}$ but high solubilities in lipid phases. ${ }^{8}$ Dissolved in such a heterogeneous medium PAHs will solubilise into the lipid phases and give extremely low aqueous concen- 
trations. ${ }^{9}$ After deposition of the carrier particles in the lining layer two transport processes connected in series may be of great importance to the in vivo behaviour of PAHs. The first process is the release of PAHs from their carrier particles and the second is the penetration of the hydrocarbons through the lining layer into the epithelium below. The traditional view is that a slow release of PAHs from their carrier particles is followed by a rapid absorption by alveolar macrophages or by the bronchial epithelium. This would give low liquid concentrations of PAHs in the lining layer. The lipid aqueous heterogeneity of the bronchial lining layer, however, reduces the rate of penetration of lipophilic substances such as PAHs more than a purely aqueous barrier would do. ${ }^{10}$ Furthermore, in addition to a PAH fraction firmly bound to the surface of carbonaceous particles, there is a reversibly bound PAH fraction both on carbonaceous ${ }^{11}$ and on mineral particles (P Gerde, P Scholander, IARC Conference 1987). This latter PAH fraction is likely to be released in a sharp pulse during deposition in the bronchial lining layer. Taken together these two effects suggest that a rapid release of PAHs from their carrier particles followed by a slow penetration into the bronchial epithelium results in high liquid PAH concentrations in the lining layer. PAHs are subsequently removed from the lung through various clearance mechanisms and metabolic degradation as shown both in animal experiments ${ }^{12}$ and in necropsies. ${ }^{13}$

A hypothetical interaction between inhaled mineral fibres and PAHs should therefore be looked for in the bronchial lining layer since this process would occur between the fibres and the dissolved fraction of PAHs. The equilibrium liquid adsorption is then a parameter of great importance.

The adsorption of PAHs, mostly BaP, on to asbestos fibres in various organic solvents, ${ }^{14}{ }^{17}$ the adsorption of $\mathrm{BaP}$ on to different mineral fibres in aqueous solution at one liquid concentration, ${ }^{18}$ and the rate of mass transfer of $\mathrm{BaP}$, precoated on to various particulates including asbestos fibres, to an aqueous suspension of phospholipid vesicles have been studied. ${ }^{19}$ The starting point of the latter experiment, precipitation of PAHs especially on to mineral fibres, has been criticised as being unnatural.$^{20}$ This criticism seems justified in view of the rather weak adsorption obtained with PAHs on to mineral fibres in a humid gas phase (P Gerde, P Scholander, IARC Conference 1987). Also, owing to the low gas phase concentrations of PAHs in the ambient air, adsorption before inhalation would be so slow that these PAHs are unlikely to come from the smoker's own smoke. Consequently, adsorption in the ambient air would give the smoker as well as the non-smoker roughly the same fibre-borne dose of PAHs. The reverse process would therefore be of greater interest - that is, the release of the PAHs from their original carrier particles in the lung followed by their transfer via solution on to the mineral fibres. The adsorption equilibrium of PAHs between the mineral fibres and the surrounding solution would indicate the probability that such a transport process occurs at all.

The phospholipid fraction of lung surfactant has a strong affinity for asbestos fibres. ${ }^{21} 22$ The phospholipids are adsorbed in continuous bilayers along the fibres with their hydrocarbon tails towards each other and their polar heads directed towards both the fibre surface and the aqueous solution. ${ }^{21}$ It is doubtful whether this is to be regarded as a true adsorption, however. For materials similar to asbestos, such as oxidised silicone, the function of the surface has been described as constituting a solid support for the lipid bilayer. ${ }^{23}$ There is also a film of water, one or a few molecules thick, between the solid surface and the lipid bilayer. The physical nature of these supported phospholipid bilayers is similar to that of bilayers of the free micellar liposomes. There are three phases involved when mineral fibres are stirred up in a suspension of phospholipids in water and there should be interfacial couplings between fibre and water and between water and lipid. The connection between the lipid phase and the fibres, however, should be shielded by the thin layer of water previously mentioned. If PAHs are introduced into this system there will be one equilibrium of PAHs between the lipid and the aqueous phases with a strong phospholipid solubilisation of aromatic hydrocarbons and one adsorption equilibrium of PAHs between the fibre surfaces and the depleted water phase. These relations may be depicted schematically as shown in fig $1 \mathrm{~b}$, with the simpler analogue of a homogeneous liquid in fig la. The direct adsorption of PAHs on the fibres has to compete with the strong solubilisation of PAHs into the lipid phase and with the shielding of the fibre surfaces by the adsorbed phospholipid bilayers. The aim of this work is thus to study the equilibrium adsorption of $\mathrm{BaP}$ on to asbestos fibres and MMMF in aqueous solution and how this adsorption is influenced by the presence of phospholipids from lung surfactant.

\section{Materials and methods}

The adsorption of $\mathrm{BaP}$ was measured on three types of asbestos, chrysotile, antophyllite, and amosite, and on three types of MMMF, rock wool, slag wool, and glass wool. The joint adsorption of $\mathrm{BaP}$ and phosphatidylcholine (PC) on to chrysotile and amosite and on to rock wool and glass wool was also studied. PC was chosen as being the most common phospholipid in lung surfactant. ${ }^{24}$ Before use, all fibre materials were washed in toluene for eight hours by means of Soxhlet 


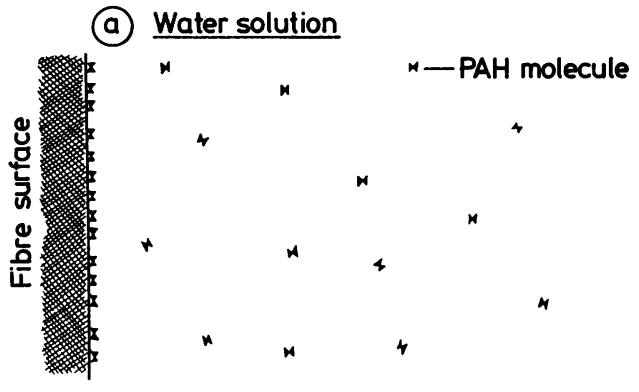

(b) Phospholipid suspension

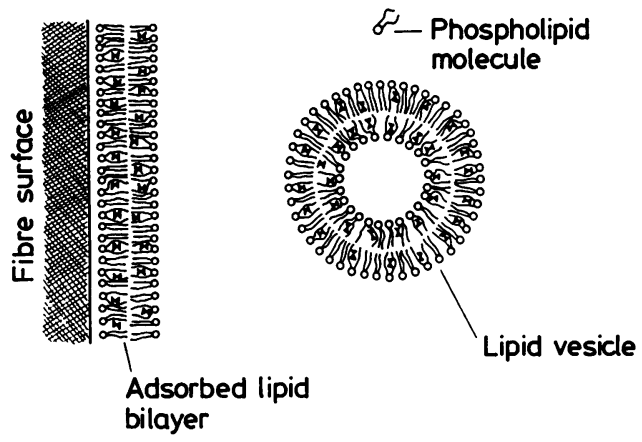

Fig 1 (a) Schematic description of equilibrium between mineral fibre surface and $P A H$ in homogeneous aqueous solution. A certain fraction of hydrocarbon is adsorbed on to fibre surface. (b) Schematic description of equilibrium between mineral fibre surface and PAH in heterogeneous phospholipid-water suspension. Phospholipid adsorbs on to fibre surface but there may be a thin layer of water, perhaps one or a few molecules thick, between lipid bilayer and fibre surface. $P A H$ is readily solubilised into both adsorbed and free lipid phases.

extraction and dried at $120^{\circ} \mathrm{C}$ for two hours to remove any trace organics on the fibres although none of the MMMF was coated with binders. The slag wool sample had to be stirred up in water and decanted in order to separate larger non-fibrous particles originating in the manufacturing process. Coagulation of PC may be caused by the leaching of magnesium from chrysotile asbestos, so this material was leached with water by Soxhlet extraction for about 24 hours. At the high ratio of fibres to surrounding medium used in the experiments, these washing procedures were necessary to obtain a fairly inert surface that would not disturb the liquid phase too much. Solitary micron size fibres in the lung are probably both leached and eluted rapidly in the lipid aqueous medium of the lung and released compounds should be rapidly diluted to negligible concentrations. The specific surface areas of the washed materials were determined by measuring their BET isotherms (table). ${ }^{3} \mathrm{H}$-benzo(a)pyrene (4.96
$\mathrm{GBq} / \mathrm{mg}$ ) from Amersham Corporation and $\mathrm{L}-\alpha-$ dipalmitoyl-[2-palmitoyl-1- $\left.{ }^{14} \mathrm{C}\right]$-phosphatidylcholine (2.67 MBq/mg) from New England Nuclear were used; when necessary this substance was diluted with non-labelled L- $\alpha$-phosphatidylcholine (egg yolk) from Sigma Chemicals. Using tritiated BaP with PC tagged with ${ }^{14} \mathrm{C}$ enabled the concentrations of $\mathrm{BaP}$ and $\mathrm{PC}$ to be measured simultaneously. Radioactivity was counted in a Packard Tri-Carb scintillometer using Bioflour (New England Nuclear) as a scintillation cocktail. All experiments were performed using a physiological solution according to Gamble (type II), ${ }^{25}$ and the water for this was both deionised and microfiltered. The major types of ions in this solution are $\mathrm{Na}^{+}, \mathrm{K}^{+}, \mathrm{Ca}^{2+}, \mathrm{Cl}^{2}, \mathrm{CO}_{3}{ }^{2-}, \mathrm{PO}_{4}{ }^{3-}$, and $\mathrm{CH}_{3} \mathrm{COO}$.

The adsorption was determined by means of the classic solution depletion method. The decrease in the liquid concentration of the adsorbing substance after the addition of a solid material is taken as a measure of the adsorption on to the material. The experiments were performed in Pyrex tubes with a volume of 15 or $70 \mathrm{ml}$ with teflon sealed screw caps. These tubes could be centrifuged directly to separate the fibres from the solution. A saturated solution of $\mathrm{BaP}$ was prepared by plating an excess of the hydrocarbon on to the bottom of a round flask. After the addition of water and gentle agitation, the solution was kept immobile for at least 24 hours before use. The solubility of $\mathrm{BaP}$ in the physiological solution was found to be $4.8 \mu \mathrm{g} / \mathrm{l}$ at $37^{\circ} \mathrm{C}$. The liquid volumes used were $7 \mathrm{ml}$ in the smaller tubes and $40 \mathrm{ml}$ in the larger ones, and the amounts of fibres used were 0.02-0.04 g and 0.2-3 g, respectively. The freshly prepared aqueous solutions were agitated for one hour in a thermostat controlled shaking bath at $37^{\circ} \mathrm{C}$ before the initial sampling was done. All liquid sampling was done in triplicate. After the addition of the fibre fraction, the tubes were replaced in the shaking bath. The liquid phase was sampled at different times up to 300 hours after the addition of the fibres. Before sampling the tubes were centrifuged at $1000 \mathrm{~g}$ for about 10 minutes. When measuring the adsorption of BaP in the physiological solution, a

\section{Specific surface areas of the fibrous materials studied}

\begin{tabular}{ll}
\hline Fibre type & $\begin{array}{l}\text { Specific } \\
\text { surface } \\
\text { area } \\
\left(\mathrm{m}^{2} / \mathrm{g}\right)\end{array}$ \\
\hline Chrysotile-B asbestos (UICC) & $40 \cdot 1$ \\
Antophyllite asbestos (UICC) & $19 \cdot 6$ \\
Amosite asbestos (UICC) & $12 \cdot 8$ \\
Glass wool (AB Gulliber, Billesholm, Sweden) & $0 \cdot 717$ \\
Rock wool (Rockwool AB, Skövde, Sweden) & $0 \cdot 275$ \\
Slag wool (Cape Insulation Limited, Middlesbrough, & $0 \cdot 174$ \\
UK) & \\
\hline
\end{tabular}

UICC $=$ Union Internationale Contre le Cancer. 


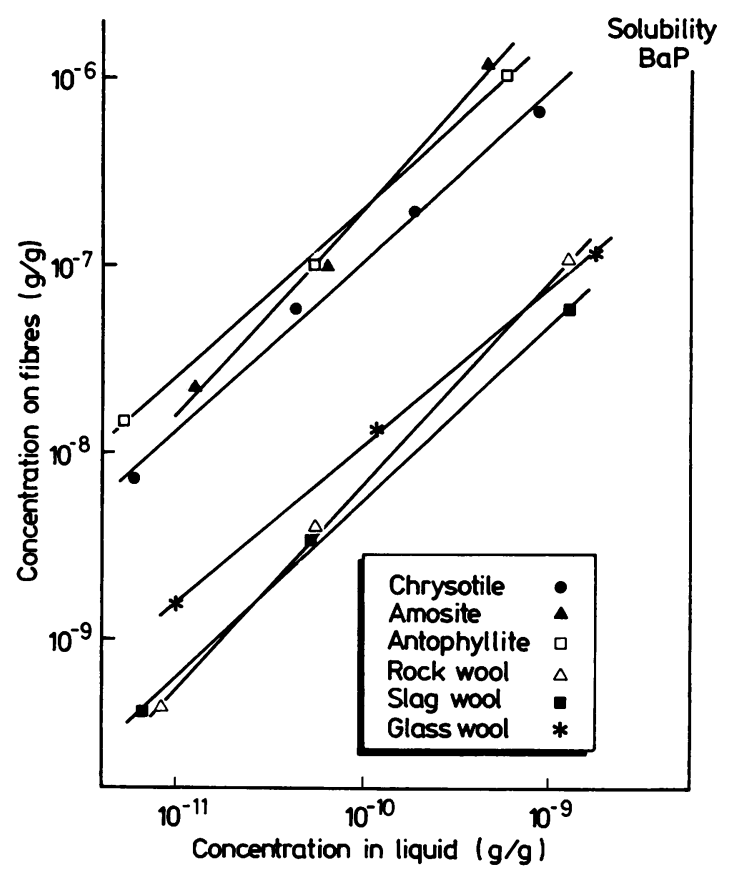

Fig 2 Adsorption isotherms of benzo(a)pyrene (BaP) on to mineral fibres in physiological water solution (Gamble-II) at $37^{\circ} \mathrm{C}$.

correction has to be introduced for a slight adsorption on to the walls of the experimental vessel.

The experiments with both $\mathrm{BaP}$ and $\mathrm{PC}$ were performed only in the larger tubes and at $41^{\circ} \mathrm{C}$ to ensure a chain melting temperature for this particular phospholipid. ${ }^{26}$ The lipid mixture of true lung surfactant has a lower chain melting temperature and is thus in a liquid state already at $37^{\circ} \mathrm{C}$. This $4^{\circ}$ increase in temperature has probably only a limited influence on the positions of the studied adsorption equilibria but it is necessary to ensure the rapid spreading of the phospholipids that has been observed in vivo. For each material the adsorption of both $\mathrm{BaP}$ and $\mathrm{PC}$ was measured simultaneously in series with constant conditions as regards liquid volume, amount of fibres added, and initial concentration of BaP but with an increasing amount of $\mathrm{PC}$ added. The initial concentration of BaP was that of the saturated aqueous solution at refrigerator temperature or about $3.5 \mu \mathrm{g} / \mathrm{l}$. The liquid volume was $40 \mathrm{ml}$ and the amount of fibres added in each series of amosite, chrysotile, and rock wool was $0 \cdot 1,0 \cdot 1$, and $2 \cdot 0 \mathrm{~g}$, respectively. Both isotopes were counted simultaneously in each sample of the liquid phase.

\section{Results and discussion}

BaP IN AQUEOUS SOLUTION

The adsorption isotherms of $\mathrm{BaP}$ at $37^{\circ} \mathrm{C}$ on to the various fibrous materials in a physiological water solution (Gamble-II) are shown in fig 2. The adsorption is calculated from several liquid concentration measurements between 50 and 100 hours after addition of the fibres. During this period the liquid concentrations were fairly stable for all materials tested. The affinity of the aromatic hydrocarbon for the tested materials is not very strong and all isotherms are close to linear. The adsorption per BET surface area is uniform and lies within the same order of magnitude for all materials tested. Even at a liquid concentration of $10 \%$ of that of a saturated solution of $\mathrm{BaP}$ there will be only a highly scattered monomolecular layer on the fibre surfaces. This weak adsorption of aromatic hydrocarbons in aqueous solutions has been reported both with asbestos ${ }^{18}$ and with other mineral particles. ${ }^{27}$ This has been explained by a competition between water molecules and the hydrocarbons for the same polar sites on the mineral surfaces. ${ }^{27} 28$

\section{BAP AND PC IN AQUEOUS SOLUTION}

The change in the liquid concentration of $\mathrm{BaP}$ and $\mathrm{PC}$ at $41^{\circ} \mathrm{C}$ as a function of time after the addition of a predetermined amount of fibres is shown in fig 3. Both $\mathrm{BaP}$ and PC concentrations before the addition of fibres were set to $1 \cdot 0$. Each fibrous material has been tested in a series with constant liquid volume, amount of fibre added, and initial concentration of $\mathrm{BaP}$ but with increasing initial PC concentration. Each of the numbers to the right in the figure identifies one experimental batch and describes the amount of PC added to this particular liquid fibre mixture. This amount of PC is expressed as a fraction of the amount needed to cover the fibre fraction with a monomolecular layer of this phospholipid. A surface area per PC molecule of $60 \mathrm{~A}^{2}$ has been assumed. ${ }^{22}$ The zero value of no $\mathrm{PC}$ added is associated only with a $\mathrm{BaP}$ concentration curve and refers to a homogeneous aqueous solution as in fig la. The other numbers describe the situation in fig $1 \mathrm{~b}$ and are thus associated with both a $\mathrm{BaP}$ and a PC concentration curve. The general trend in these experiments is repeatable not only with amosite with its set of ordered concentration curves (fig 3a) but also in the case of the drifting curves of chrysotile (fig $3 b$ ) and rock wool (fig $3 c$ ).

Although it may look somewhat confusing, there are three important reasons for showing one set of concentration curves for each of the major types of fibres tested. Firstly, it is evident that all fibrous materials tested adsorb PC and secondly, in the case of both the MMMF (fig 3c) and chrysotile asbestos (fig 
(a)

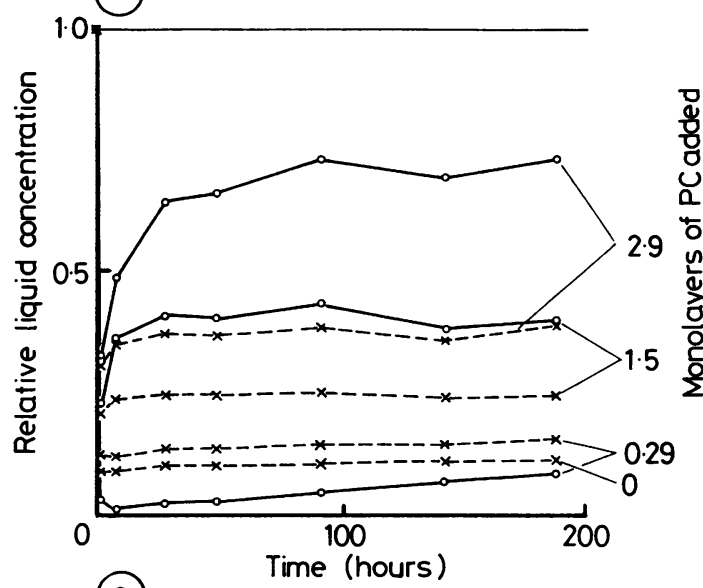

(C)

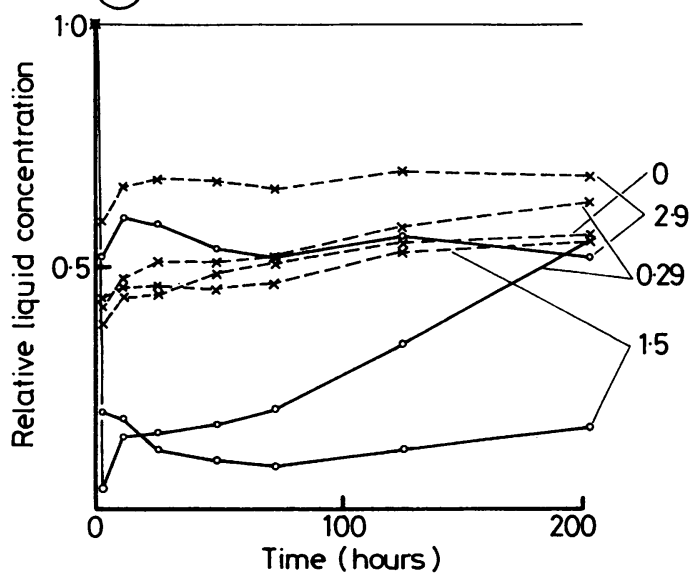

(b)

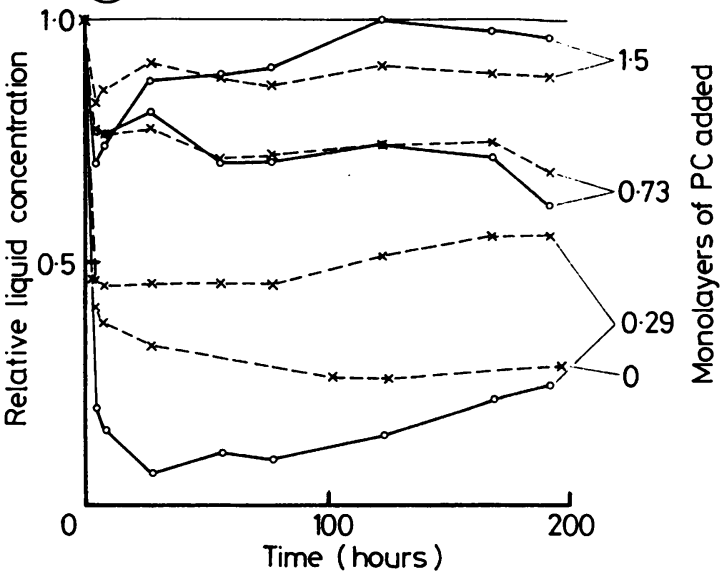

$\multimap$ Phosphatidylcholine (PC)

$x---x$ Benzo (a)pyrene (BaP)

Fig 3 Joint adsorption of BaP and phosphatidylcholine (PC) on to mineral fibres in a physiological water solution (Gamble-II) at $41^{\circ} \mathrm{C}:$ (a) amosite asbestos, (b) chrysotile asbestos, (c) rock wool. Relative decrease in liquid concentration of $B a P$ and $P C$ on addition of predetermined amount of fibres is shown. Each material has been tested in a series with constant parameters as regards liquid volume, amount of fibre added, an initial concentration of BaP but with an increasing amount of $P C$ added. Numbers on right indicate amount of $P C$ added to each experimental batch and this is expressed in how many monolayers this amount of $P C$ can form on particular portion of fibre in this tube.

$3 b)$, this adsorption is not stable but changes with time. By contrast, the amphibolic asbestos amosite has a much more stable adsorption of PC (fig 3a), a condition that probably mirrors the greater stability of this material in water compared with the others. ${ }^{29} \mathrm{~A}$ third important conclusion may be drawn from these results: without $\mathrm{PC}$ the curve of the $\mathrm{BaP}$ concentration represents the direct adsorption of this hydrocarbon on to the fibre surfaces (fig 1A). When PC is present in the system, however, both the concentration in the aqueous phase and the direct adsorption of $\mathrm{BaP}$ on to the fibres will be negligible owing to the large lipid aqueous partition coefficient of $\mathrm{BaP}(\mathrm{fig} 1 \mathrm{~b})$. This may be shown quite easily. By means of solubility measurements we have estimated the partition coefficient of BaP between PC and water to be about $10^{7}$. Even the amount of PC corresponding to the lowest concentration added in the experiments will cause a drop in the water concentration of $\mathrm{BaP}$ to about one promille of the average concentration in this sample. This number may be inserted into the linear adsorption isotherms of the aqueous solution (fig 2) in order to calculate a hypothetical amount of $\mathrm{BaP}$ adsorbed by fibres in a lipid aqueous solution undisturbed by lipid adsorption. If this amount of $\mathrm{BaP}$ is compared with the total amount added to the vessel it 
is evident that the direct adsorption of $\mathrm{BaP}$ on to the fibres would be too weak to be detected. It must therefore be concluded that the decrease in the concentration of $\mathrm{BaP}$ that is actually detected when PC is present in the system is caused by the solubilisation of $\mathrm{BaP}$ into layers of $\mathrm{PC}$ that in turn are adsorbed on to the fibres. This view is further supported by a definite connection between the concentrations of $\mathrm{BaP}$ and $\mathrm{PC}$ in the same vessel. Coinciding curves of these concentrations indicate that $\mathrm{BaP}$ has roughly the same affinity for lipid phases adsorbed on fibres as for those in the free micellar liposomes. If the curve of the $\mathrm{BaP}$ concentration lies below that of the PC concentration this means that $\mathrm{BaP}$ has a greater affinity for adsorbed lipids than for the free lipid phases and vice versa. For example, addition of the fraction of amosite asbestos to the batch with 1.5 monolayers of PC (fig 3a) causes a $75 \%$ reduction in the liquid concentration of $\mathrm{BaP}$ and a $60 \%$ reduction in the liquid concentration of $\mathrm{PC}$. Seventy-five percent of the BaP fraction solubilises into the $60 \% \mathrm{PC}$ that is adsorbed on to the fibres, leaving $25 \% \mathrm{BaP}$ in the remaining $40 \% \mathrm{PC}$ in the solution. Thus the concentration of $\mathrm{BaP}$ in lipids adsorbed on fibres is in this case twice as high as the $\mathrm{BaP}$ concentration in the free liposomes.

The solution depletion method is not suitable for the exact estimation of the adsorption of phospholipids on to fibres in the lung. The maximum possible ratio of PC to fibres is still far too low to simulate true conditions around a single fibre in the lung. Amosite asbestos may be mentioned as an example. According to fig $3 \mathrm{a}$ this material adsorbs 0.8 to 0.9 monomolecular layers of PC. Direct surface analysis at an excess of PC, however, indicated a full bilayer of phospholipids on the fibres. ${ }^{2122}$

We conclude that the direct adsorption of PAHs on to mineral fibres in the lung is negligible. Moreover, small amounts of aromatic hydrocarbons adsorbed in the ambient air before inhalation (P Gerde, P Scholander, IARC Conference 1987) are likely to be released rapidly after pulmonary deposition. ${ }^{20} \mathrm{~A}$ subsequent strong adsorption of surfactant phospholipids on to the fibres however, may create a continuous lipid phase along the fibre surface, within which lipophilic substances such as PAHs will be solubilised and are likely to have a high mobility. This may create lipid connections across aqueous regions otherwise impermeable to lipophilic substances. ${ }^{30}$ This could be of particular importance in the bronchial lining layer with its highly structured distribution of lipid and aqueous phases. ${ }^{6}$ These results have led us to formulate a new hypothesis to explain the mechanism behind the synergistic effect observed between tobacco smoking and exposure to asbestos for the induction of bronchial cancer.$^{30}$ Phospholipid adsorption by the fibres and not their direct adsorption of aromatic hydrocarbons should therefore be the crucial parameter for this indirect interaction between fibres and aromatic hydrocarbons.

This work was supported by the Swedish Work Environment Fund through grant No 81-0929.

\section{References}

1 Selikoff IJ, Hammond EC, Churg J. Asbestos exposure, smoking and neoplasia. JAMA 1968;204:106-12.

2 Harington JS. Chemical factors (including trace elements) as etiological mechanisms. In: Bogovski P, Gilson JC, Timbrell V, Wagner JC, eds. Biological effects of asbestos. Lyon: International Agency for Research on Cancer, 1973:304-11. (Sci pub No 8.)

3 Morgan A, Evans JC, Evans RJ, Hounam RF, Holmes A, Doyle SG. Studies on the deposition of inhaled fibrous material in the respiratory tract of the rat and its subsequent clearance using radioactive tracer techniques. Environ Res 1975;10:196-207.

4 Lippmann M, Schlesinger RB. Interspecies comparisons of particle deposition and mucociliary clearance in tracheobronchial airways. J Toxicol Environ Health 1984;3:441-69.

5 Lopez-Vidriero MT, Reid L. Respiratory tract fluid - chemical and physical properties of airway mucus. Eur $J$ Respir Dis 1980;61(suppl 110):21-6.

6 Ueda S, Kawamura K, Ishii N, et al. Ultrastructural studies on surface lining layer (SLL) of the lungs: Part III. Journal of Japanese Medical Society Biological Interface 1984;15:67-78.

7 Mackay D, Shiu WY. Aqueous solubility of polynuclear aromatic hydrocarbons. Journal of Chemical Engineering Data 1977;22:399-402.

8 Patton JS, Stone B, Papa C, Abramowitz R, Yalkowsky SH. Solubility of fatty acids and other hydrophobic molecules in liquid trioleoylglycerol. J Lipid Res 1984;25:189-97.

9 Thust R, Gräbner R. Incorporation in liposomes as a method for the application of genotoxins of low water solubility in the SCE assay. Mutat Res 1984;139:207-10.

10 Gerde P, Scholander P. A mathematical model of the penetration of polycyclic aromatic hydrocarbons through a model of the bronchial lining layer. Environ Res 1987;44:321-34.

11 Rivin D, Atkins JH. Sorption of bioactive materials on carbon. Carbon 1987;27:135-45.

12 Sun JD, Wolff RK, Kanapilly GM. Deposition, retention and biological fate of inhaled benzo(a)pyrene adsorbed onto ultrafine particles and as a pure aerosol. Toxicol Appl Pharmacol 1982;65:231-44.

13 Brockhaus A, Tomingas R, Dehnen W, Pott F, Beck EG. Das verhalten kanzerogener kohlenwasserstoffe in der lunge. Praxis der Pneumologie 1971;25:519-26.

14 Shabad LM, Pylev LN, Krivosheeva LV, Kulagina TF, Nemenko BA. Experimental studies on asbestos carcinogenicity. $J$ Natl Cancer Inst 1974;52:1175-80.

15 Harington JS, Smith M. Studies on hydrocarbons on mineral dusts. Arch Environ Health 1964;8:453-8.

16 Fournier J, Pezerat H. Studies on surface properties of asbestos. Environ Res 1986;41:276-95.

17 Menard H, Noel L, Khorami J, Louve J-L, Dunnigan J. The adsorption of polyaromatic hydrocarbons on natural and chemically modified asbestos fibres. Environ Res 1986;40:84-91.

18 Harvey G, Pagé M, Dumas L. Binding of environmental carcinogens to asbestos and mineral fibres. Br J Ind Med 1984;41: 396-400.

19 Lakowicz JR, Bevan DR, Riemer SC. Transport of a carcinogen, benzo(a)pyrene, from particulates to lipid bilayers. A model for the fate of particle-adsorbed polynuclear aromatic hydrocarbons which are retained in the lungs. Biochim Biophys Acta 
1980;629:243-58.

20 Chang MJW, Singh NP, Turturro A, Hart RW. Interaction of benzo(a)pyrene and chrysotile. In: International symposium on polynuclear aromatic hydrocarbons 6. Columbus 1981. Columbus, Ohio: Battelle Press, 167-76.

21 Light WG, Wei ET. Surface charge and hemolytic activity of asbestos. Environ Res 1977;13:135-45.

22 Jaurand MC, Thomassin JH, Baillif P, Magne L, Touray JC, Bignon J. Chemical and photoelectron spectrometry analysis of the adsorption of phospholipid model membranes and red blood cell membranes on to chrysotile fibres. $\mathrm{Br} J$ Ind Med 1980;37:169-74.

23 Tamm LK, McConnell HM. Supported phospholipid bilayers. Biophys J 1985;47:105-13.

24 Schlimmer P, Austgen M, Ferber E. Classification and possible function of phospholipids obtained from central airways. Eur $J$ Respir Dis 1983;64(suppl 128):318-21.

25 Gamble JL. Chemical anatomy, physiology and physiology of extracellular fluid. 8th ed. Boston: Harvard University Press, 1967.

26 Clements JA. Functions of the alveolar lining layer. Am Rev Respir Dis 1977;115:67-71.

27 Chiou CT, Shoup TD. Soil sorption of organic vapors and effects of humidity on sorptive mechanism and capacity. Environmental Sciences Technology 1985;19:1196-1200.

28 Fournier J, Pezerat H. Etude de mode d'adsorption des hydrocarbures polycycliques aromatiques sur les amiantes. Cas du phenantrene. Journal de Chimie Physique 1982;79:589-96.

29 Morgan A, Holmes A. The deposition of MMMF in the respiratory tract of the rat, their subsequent clearance, solubility in vivo and protein coating. In: Proceedings of the conference biological effects of man-made mineral fibres, Copenhagen, 1982. Vol 2. Copenhagen: WHO/IARC, 1982:1-17.

30 Gerde $P$, Scholander $P$. A hypothesis concerning asbestos carcinogenicity: the migration of lipophilic carcinogens in adsorbed lipid bilayers. Ann Occup Hyg 1987;31:395-400. 